\title{
Deep Contextual Bandits for Fast Neighbor-Aided Initial Access in mmWave Cell-Free Networks
}

\author{
Insaf Ismath, Samad Ali, Nandana Rajatheva, and Matti Latva-aho \\ Center for Wireless Communication, University of Oulu, Oulu, Finland \\ \{insaf.ismath, samad.ali, nandana.rajatheva, matti.latva-aho\}@oulu.fi
}

\begin{abstract}
Access points (APs) in millimeter-wave (mmWave) user-centric (UC) networks will have sleep mode functionality. Initial access (IA) is a challenging problem in UC networks due to the coherent serving of the users. In this paper, a novel deep contextual bandit (DCB) learning-based instantaneous beam selection method is proposed as a complementary tool to current IA schemes. In the proposed approach, the DCB model at an AP uses beam selection information from the neighboring active APs as the input to solve the beam search problem of the host AP. The proposed fast beam selection scheme enables APs to be in energy-saving modes while maintaining the ability to serve users without any delay when restored. Simulations are carried out with realistic channel models generated using a ray-tracing tool. The results show that the proposed system with the 5G IA scheme can respond to dynamic throughput demands with negligible latency compared to the $5 \mathrm{G}$ IA scheme without the proposed scheme.
\end{abstract}

Index Terms-Initial access, mmWave, deep contextual bandits, user-centric, deep reinforcement learning.

\section{INTRODUCTION}

To facilitate applications like extended reality (XR) that demand high throughput low-latency communication, networks of the future are expected to provide at least a 1000-fold increase in network throughput [1]. Despite high sensitivity to blockages, millimeter wave (mmWave) and sub-THz bands will be exploited to attain throughput goals [2]. Massive multiple-input multiple-output (MIMO) enabled user-centric (UC) topology is proposed to provide a uniform quality of service throughout the coverage area. It is proposed as an alternative to the conventional cellular system where the coverage area is divided into cells, each served by a base station. The UC architecture proposes to distribute sets of antennas throughout the coverage area in the form of access points (APs) instead of placing them centrally in a base station similar to cellular systems. Users are served by coherent joint transmissions made by multiple APs. Uniform quality of service is ensured with a dense deployment of APs throughout the coverage area. However, all the APs might not be required all the time to provide a good quality of service [3]. Therefore, maintaining a network with a large number of otherwise redundant APs causes considerably inefficient power usage.

Power consumption can be improved by putting the redundant APs into a reduced functionality mode called the sleep-state that consumes less power [3], [4]. With highly dynamic mmWave channels, users may suddenly lose connections to some APs [2]. Furthermore, users may instantly need additional radio resources to begin or maintain high throughput low-latency applications. Networks of the future are expected to maintain a consistent quality of service against such impromptu changes by promptly rearranging the network, i.e., the state of the APs. To do so, restored sleep-state APs are required to contribute to the network capacity instantly. Inability to achieve this would cause an additional latency component. A recently restored AP, like any other AP, has to perform initial access (IA). Among other things, periodically performed IA is responsible for identifying the best beam to serve a user. Although strategies were presented to improve energy efficiency, the beam search problem in IA is not considered in [3], [4]. The 5G IA uses an exhaustive search-based method called beam-sweeping to perform the beam search [5]. Works like [6], [7] provide algorithms to improve the beam search time. Authors in [8] present a machine learning (ML) based strategy to provide faster IA. However, these approaches require interacting with the user. These interactions are performed inside a periodically occurring IA window in the radio frame. Due to the coherent user serving in the UC system, these radio frames must be synchronized to avoid interference to the ongoing communications of the user [9]. Therefore, a restored sleep-state AP has to wait for the next shared IA window causing a significant delay.

To address this gap, we propose a novel beam selection approach that does not require user responses. Here the beam selection problem at a recently restored AP is formulated involving its neighboring active APs. The proposed approach uses beam choices made by the neighboring APs to serve a user as a crude representation of the environment between the APs and the respective user at that instance. Then this representation is mapped into one of the beams of the recently restored AP's beam codebook. Beam resulting from this mapping is used by the restored AP to serve the respective user. Therefore, the recently restored AP is not required to wait for the next IA window. Since this mapping function is complex to model, a deep neural network (DNN) is used to approximate it. The deep contextual bandit (DCB) architecture is used to explore and learn this mapping function.

The proposed method provides instantaneously beam selection for recently restored APs irrespective of the location at the radio frame. However, the proposed approach is meant to be deployed alongside a periodic stand-alone IA scheme. The performance of this neighbor-aided IA system is evaluated using realistic scenarios generated using a ray-tracing tool. Simulation results for the considered scenario show that the instantaneous predictions of the DCB model achieve $94 \%$ of 


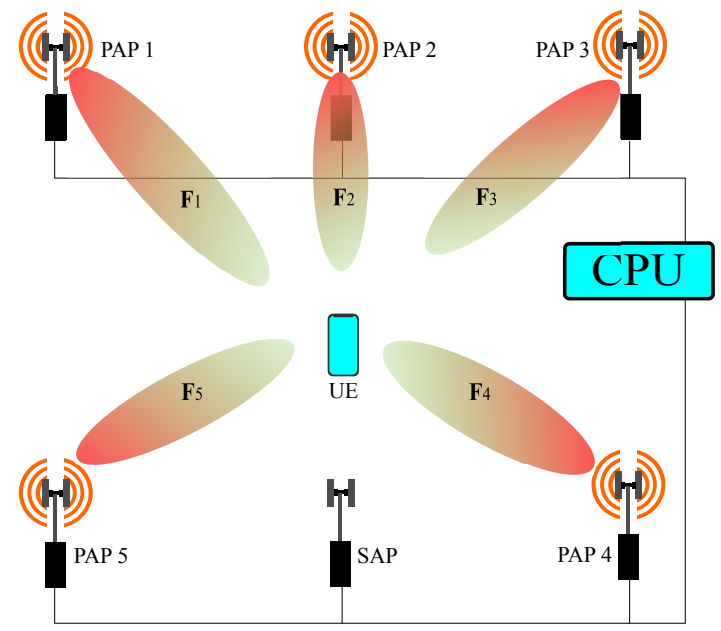

Fig. 1: The system model of a UC network where a set of APs would simultaneously serve a single user.

the best case signal-to-noise ratio (SNR) while maintaining a negligible latency.

The rest of this paper is organized as follows. Section II explains the system model used in this work and introduces the IA problem. Section III formally presents the proposed approach and provides a quick primer on DCB. Section IV explains details about the simulations and presents numerical results and Section $\mathrm{V}$ concludes the paper.

Notations: $(.)^{T}$, and $(.)^{H}$ denote transpose and Hermitian transpose, respectively. $\|\mathbf{x}\|$ and $|\mathbf{x}|$ denote the euclidean norm and cardinality of $\mathbf{x}$, respectively. $\mathbb{1}(\mathbf{x})$ is a sparse matrix where each row $i$ presents the one-hot encoding for $i$ th element of the row vector $\mathbf{x} .\{\mathbf{X}\}$ represents the vector space containing all possibilities for $\mathbf{X}$.

\section{System Model and Problem Formulation}

\section{A. System Model}

Consider a UC network with $N$ densely deployed APs and one user as presented in Fig. 1. APs are equipped with uniform rectangular planar antenna arrays (URPA) with $M$ antenna-elements. Here, the single antenna user is coherently served by multiple APs to ensure reliability and to maintain high throughput communication. All APs are connected to their controlling entity, the central processing unit (CPU) via backhaul links. To conserve energy APs can operate in two power consumption modes: the fully functional awake-state and the reduced functionality power-saving sleep-state [3], [4]. APs in the awake-state and sleep-state are defined as primary APs (PAPs) and secondary APs (SAPs), respectively. Work in [3], [4] and others improve network power consumption by providing PAP selection strategies. However, for the sake of simplicity, it is assumed that the CPU randomly selects $\bar{N}$ APs as PAPs and one AP as an SAP. The capacity provided by this configuration is assumed to satisfy the off-peak throughput requirement of the network. When the throughput demand of the network surpasses the current network capacity, the CPU decides to restore the SAP. Each active AP periodically performs a stand-alone IA scheme in addition to the proposed beam search approach. The mmWave channel is modeled with a clustered model [10]. Each channel is generated using $K$ clusters which are made combining $Q$ paths characterized by path loss, fading, and array gain at the AP. The subscript notations $k=1, \ldots, K$ and $q=1, \ldots, Q$ represent cluster and path index, respectively. The channel from an AP to the user $\mathbf{h} \in \mathbf{C}^{1 \times M}$ is presented as

$$
\mathbf{h}=\frac{1}{\sqrt{Q}} \sum_{k=1}^{K} \sum_{q=1}^{Q} p_{k, q} r_{k, q} \mathbf{a}\left(\theta_{k, q}, \phi_{k, q}\right),
$$

where $p_{k, q} \in \mathbf{C}$ and $r_{k, q} \in \mathbf{C}$ represent the path loss and small-scale fading gain, respectively, and $\mathbf{a}\left(\theta_{k, q}, \phi_{k, q}\right) \in$ $\mathbf{C}^{1 \times M}$ is the AP's array gain. Here $\theta_{k, q}$ and $\phi_{k, q}$ are the azimuth and elevation angles of arrival at the AP, respectively. The channels used in this work are generated using a raytracing tool called Wireless InSite [11] to capture realistic mmWave behaviors.

The authors of [12] suggest using analog or hybrid beamforming techniques for mmWave MIMO communications to relax the power and hardware requirements imposed by the usage of fully digital systems. Hence, this work only considers analog beamforming which is implemented using an array of $M$ quantized phase shifters. Beamforming codebook $\mathbf{F}$ is the finite set of $M$ beams generated using this arrangement and the $i$ th beam $\mathbf{f}_{i} \in \mathbf{F}$ is $\frac{1}{\sqrt{M}}\left[e^{j \Theta_{i, 1}} \ldots e^{j \Theta_{i, M}}\right]^{T}$. Here $\Theta_{i, m}$ is the phase shift added to the signal emitted from the $m$ th antenna-element corresponding to the $i$ th beam.

\section{B. Problem Formulation}

During IA beam search APs have to identify the beam which maximizes the received SNR at the user. The SNR component provided by the $n$th PAP to the user is $\Gamma_{n}$ and it is expressed as

$$
\Gamma_{n}\left(b_{n}\right)=\frac{P_{n}\left\|\mathbf{h}_{\mathbf{n}} \mathbf{f}_{b_{n}}\right\|^{2}}{\sigma^{2}},
$$

where $P_{n}$ is the transmit power of $n$th PAP, $\sigma^{2}$ is the noise power at the user, $\mathbf{h}_{\mathbf{n}}$ is the channel $\mathbf{h}$ from $n$th PAP to the user, and $b_{n}$ and $\mathbf{f}_{b_{n}}$ represent the beam index and the corresponding beam chosen by $n$th PAP to serve the user, respectively. The IA problem at $n$th PAP is presented as

$$
\begin{array}{cl}
\underset{b_{n}}{\operatorname{maximize}} & \Gamma_{n} \\
\text { subject to } & b_{n} \in\{1, \ldots M\} .
\end{array}
$$

The 5G IA scheme periodically solves (3) at each AP using a beam-sweep [?]. This beam searching mechanism involves the sequential transmission of beams in $\mathbf{F}$. With each beam, a synchronization signal block (SSB) which spans over 20 resource blocks across 4 OFDM symbols is transmitted. SSBs provide system information, radio frame synchronization and allows users to measure the received signal power. Beams providing the best measurements are reported to the respective APs. Apart from the 5G standard, the work in [6]-[8] present methods to provide faster IA. However, all of these IA schemes solving the general beam search problem require some shape or form of user interactions. In the UC setting, such IA 
processes in serving APs must occur in a common IA window to avoid disrupting coherent transmissions. Hence, when an SAP is restored it is forced to wait until the next shared IA window to identify the serving beam to serve the user.

\section{DCB BASED NEIGHBOR-AIDED INITIAL ACCESS APPROACH}

\section{A. Proposed Approach}

All the APs and the user share the same close physical environment. Therefore, at least some of the channels between APs and the user are not completely statistically independent leading to a degree of spatial correlation. The beams selected by APs can be taken as crude representations of their respective channels with the user. When considered as a whole, this set of beams can be used as a rough state representing the physical environment. In theory, one could hope to find a function which maps this state to the beam codebook of an AP in the same close physical environment with some degree of error.

With the proposed approach, an SAP approximates the achievable SNR at the user for all $\mathbf{f}_{i} \in \mathbf{F}$ using the mapping function $\mathbf{g}(\mathbf{L}, \mathbf{B})$ which is defined as

$$
\mathbf{g}(\mathbf{L}, \mathbf{B}):\{\mathbf{L}\} \times\{\mathbf{B}\} \rightarrow\left\{\frac{P_{0}\left\|\mathbf{h}_{\mathbf{0}} \mathbf{f}_{i}\right\|^{2}}{\sigma^{2}}\right\}, \forall \mathbf{f}_{i} \in \mathbf{F},
$$

where $\mathbf{L}=\left[\mathbf{l}_{1} \ldots \mathbf{l}_{\bar{N}}\right]^{T}, \mathbf{B}=\left[b_{1} \ldots b_{\bar{N}}\right]$, and the familiar terms with 0th index correspond to the quantities of the SAP. Here $\bar{N}$ is the number of PAPs neighboring the SAP and $\mathbf{l}_{n} \in \mathcal{R}^{1 \times 2}$ contains latitude and longitude coordinates of $n$th PAP. The SAP selects the serving beam $\mathbf{f}_{b_{0}}$ such that

$$
b_{0}=\underset{i \in\{1, \ldots M\}}{\operatorname{argmax}} \mathbf{g}(\mathbf{L}, \mathbf{B}) .
$$

This formulation presents the SAPs' IA beam search problem as a function of $\mathbf{L}$ and $\mathbf{B}$, and therefore, enables an SAP to identify the serving beam without interacting with the user. However, mathematical characterization of $\mathbf{g}(\mathbf{L}, \mathbf{B})$ is complex due to the plethora of phenomena affecting the radio environment such as fast fading. Therefore, a DNN is used to approximate $\mathbf{g}(\mathbf{L}, \mathbf{B})$. Authors of [13] use a DNN to approximate a similarly complex but different function which maps sub- $6 \mathrm{GHz}$ channel measurements in an AP to mmWave beams in the same AP to solve the general beam search problem. Unlike [13], this work solves a beam search problem solely based on some information collected from the neighbors, and therefore, does not require dual-band deployment at the APs. Furthermore, the proposed approach solves the presented latency problem which is unique to IA of SAPs in the UC architecture. Hence, the problem and context considered in this work are different from the general beam search problem and the context of [13].

The user is already connected to the network (the CPU) via other serving PAPs. Therefore, it is assumed that the relevant synchronization-related configurations could be resolved by the CPU and shared with the SAP. The proposed approach enables recently restored SAPs to identify the serving beams instantly without involving the user. However, the proposed approach is not meant as an alternative for a stand-alone IA method, beam-sweeping-based or otherwise. It is but a complementary tool to be used alongside any periodic IA scheme. At the next IA window, all active APs, including the recently restored AP, are expected to follow the default IA scheme of the network to update their beam choices.

Message transfers between APs are kept low by saving location information of the neighbors at the APs and by sharing only beam indexes among the APs via the CPU. Therefore, a significant impact on signaling overhead is not expected.

\section{B. An Introduction to Deep Contextual Bandits (DCB)}

Contextual bandits [14] are a special case of reinforcement learning (RL) [15]. The RL architecture has a software agent that interacts with an environment by performing actions on it. The state of the environment is characterized by the context $\mathcal{X}$. Reward $\alpha_{a}$ measures the suitability of the action $a$ chosen by the agent given $\mathcal{X}$. The reward is provided to the agent as feedback on its actions. The goal of the agent is to maximize the received reward. The agent learns the $\{\mathcal{X}\} \times\{a\} \times\left\{\alpha_{a}\right\}$ space and the acquired knowledge is used to pick the action which is most likely to provide the highest reward given $\mathcal{X}$. Unlike a general RL problem, the action taken in a contextual bandit problem does not affect $\mathcal{X}$ of the environment. After receiving the actual reward, the parameters of the selection criteria are updated. However, learning $\mathcal{X}-a-\alpha_{a}$ relationships in problems having large continuous context spaces can be complex, and therefore, a DNN is used to approximate a function which predicts $\alpha_{a}$ for each action $a$ given $\mathcal{X}$ [16]. Contextual bandits with DNNs are called DCBs. The agent follows a decaying $\epsilon$-greedy exploration policy where the $\{\mathcal{X}\} \times\{a\} \times\left\{\alpha_{a}\right\}$ space is learnt by exploring the environment by taking random actions with a probability of $\epsilon$. Otherwise, the agent leverages the already acquired knowledge to choose actions such that $\alpha$ is maximized.

The DCB architecture is implemented in this work by training the DNN in the DCB model to approximate (4). Therefore, $\mathcal{X}$, actions, and $\alpha_{a}$ are defined corresponding to $\{\mathbf{L}, \mathbf{B}\}, \mathbf{F}$, and received SNR at the user, respectively. The context $\mathcal{X}$ is defined as

$$
\mathcal{X}:=\left[\frac{\mathbf{L}}{\|\mathbf{L}\|}, \mathbb{1}(\mathbf{B})\right],
$$

where $\frac{\mathbf{L}}{\|\mathbf{L}\|} \in \mathcal{R}^{\bar{N} \times 2}$ and $\mathbb{1}(\mathbf{B}) \in \mathcal{R}^{\bar{N} \times M}$. The column vector made by concatenating the columns of $\mathcal{X}$ is used as the input for the DNN. Action $a$ corresponds to the $a$ th beam in F, i.e., $\mathbf{f}_{a}$. The reward for choosing action $a$ is defined as $\alpha_{a}$ and it is presented as

$$
\alpha_{a}:=\frac{\left\|\mathbf{h}_{\mathbf{0}} \mathbf{f}_{a}\right\|^{2}}{\sigma^{2}} \times \frac{1}{\left\|\mathbf{h}_{\mathbf{0}} \mathbf{h}_{\mathbf{0}}{ }^{H}\right\|^{2}},
$$

where $\left\|\mathbf{h}_{\mathbf{0}} \mathbf{h}_{\mathbf{0}}{ }^{H}\right\|^{2}$ normalizes the performance of $\mathbf{f}_{a}$ against the conjugate beam $\mathbf{h}_{\mathbf{0}}{ }^{H}$. The performance of the DCB agent is evaluated using regret $\delta$ that benchmarks the chosen action against the best action that provides the highest reward $\alpha_{\max }$. In this work, $\delta$ is defined as

$$
\delta:=\alpha_{\max }-\alpha_{a}
$$




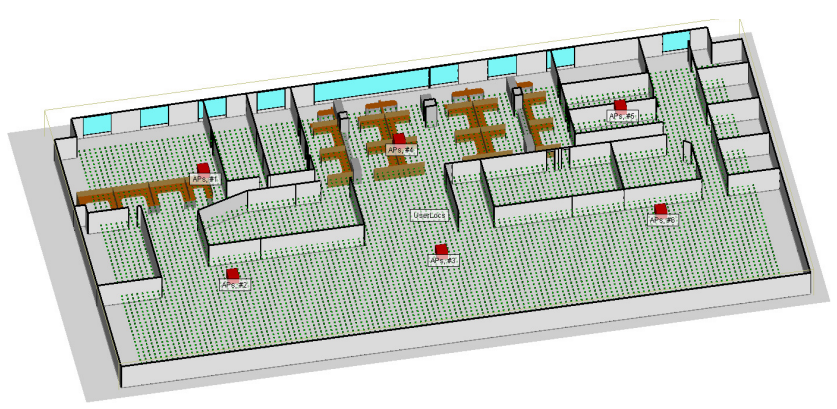

Fig. 2: The indoor office simulation environment.

\section{Simulations}

\section{A. Simulation Environment}

The indoor office environment shown in Fig. 2 is modeled with Wireless InSite ray-tracer [11] using ITU $60 \mathrm{GHz}$ compliant material models. An area of $60 \mathrm{~m} \times 30 \mathrm{~m}$ is partitioned using dry-wall into smaller office spaces. The ceiling and the floor are built with concrete. APs are located $10 \mathrm{~m}$ apart in a grid formation centering the ceiling which is $2.6 \mathrm{~m}$ above the floor. A grid of possible user locations is defined spanning the office area. All AP and possible user locations are denoted in red and green color markings in Fig. 2.

A carrier frequency of $60 \mathrm{GHz}$ and a channel bandwidth of $60 \mathrm{MHz}$ is considered. All APs are equipped with URPA antennas with 16 elements in the $4 \times 4$ configuration. The beam codebook is comprised of 16 beams. The gain and the noise figure of every antenna are set to 5 and $3 \mathrm{~dB}$, respectively. The transmit power of the APs, and the users are set to $20 \mathrm{dBm}$ and $5 \mathrm{dBm}$, respectively.

The DNN of the DCB model has 3 hidden layers each containing 100 neurons. Input layer of the DNN ingests $\mathcal{X}$ and it has $2 \bar{N}+\bar{N} M$ neurons. The output layer contains $M$ neurons corresponding to $M$ beams (actions). ReLu and Adam have been used as the layer activation function and the optimizer respectively. However, further fine-tuning of the model parameters is possible. The learning rates is set to 0.01 . In this work, $\epsilon$ is set to 1 at inception and decayed by a factor of 0.9999 every episode until a minimum of 0.01 is reached.

A simulation round consists of 50,000 episodes. At the beginning of each simulation round, one SAP and $\bar{N}$ PAPs are randomly chosen from the available APs; the user is served by all the active $\bar{N}$ APs. For the ease of simulations, the ML model is only hosted in the SAP. At each episode, the user is randomly placed in the simulation area. The proposed approach is implemented with the 5G IA scheme. However, it is not limited as such and it can be implemented with any IA scheme. All the active APs periodically perform 5G IA. An OFDM symbol duration is assumed to be $0.02 \mathrm{~ms}$, and hence, an SSB transmission takes $0.08 \mathrm{~ms}$.

The performance of the proposed approach is analyzed in terms of regret, network capacity, and normalized effective rate $\eta$ which is defined as

$$
\eta=\left(1-\frac{T_{I A}}{T_{p}}\right) \frac{\log \left(1+\Gamma_{n}\left(b_{n}\right)\right)}{\log \left(1+\Gamma_{n}^{*}\right)}
$$

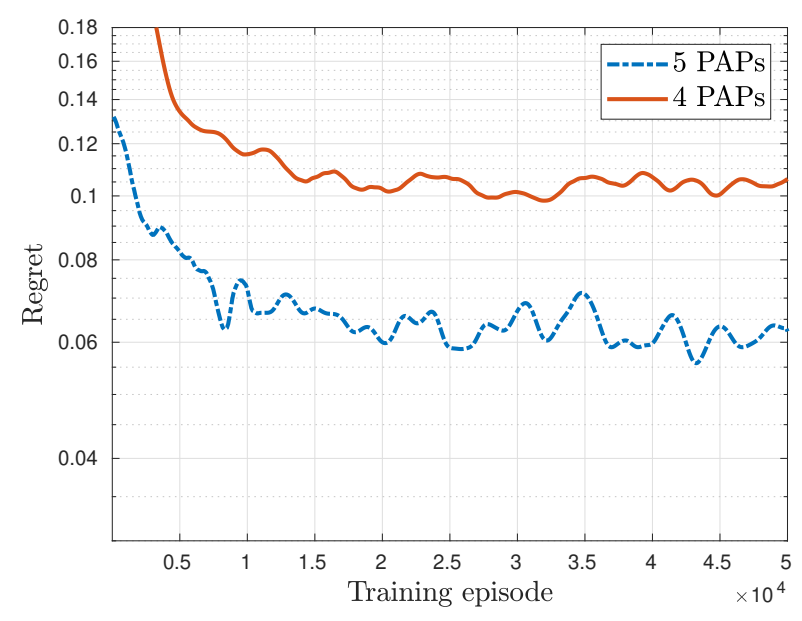

Fig. 3: Regret incurred by the DCB model during training.

where $T_{I A}$ is the time taken for the beam search, $T_{p}$ is the IA periodicity which is set to $20 \mathrm{~ms}$ by default in the $5 \mathrm{G}$ standard [17], and $\Gamma_{n}^{*}$ is the maximum achievable SNR.

\section{B. Simulation Scenarios and Results}

Fig. 3 presents the regret incurred by the DCB over training episodes for two scenarios where $\bar{N}$ is set to 4 and 5 PAPs, respectively. Due to higher number of neighbors in the 5 PAP case, the DCB model has access to more information compared to the 4 PAP case. Therefore, the DCB model in the 5 PAP case could learn faster and better compared to the 4 PAP case and results in a regret measure around 6\%.

In Fig. 4, a scenario capturing systems network capacity performance under changing user throughput demand is investigated. The SAP uses a DCB model trained with 5 PAPs. Initially, the user is served by 5 PAPs and at $t=10 \mathrm{~ms}$, an additional throughput requirement of $200 \mathrm{Mb} / \mathrm{s}$ is induced at the user. The CPU responds to this change by restoring the SAP, and therefore, user is served by 5 PAPs and an SAP after the $t=10 \mathrm{~ms}$ mark. The performance of the 5G IA method with and without the proposed approach are compared. Additionally, an always-on method where all APs are kept in the awake-state is considered. The green color area in the Fig. 4 correspond to the common IA window where the 5G IA beam-sweeps of active APs would take place. Although always-on scheme is immune to the changes in the user throughput demand, it has the least overall energy efficiency. The 5G IA with the proposed approach has enabled the SAP to start serving the user immediately. Since the $5 \mathrm{~ms}$ long IA window arrives every $20 \mathrm{~ms}$, an SAP without the proposed approach may incur an additional delay of up to $15 \mathrm{~ms}$. Although the analyzed case only has 5 PAPs and an SAP, the proposed method can be easily extended to other configurations.

By ignoring the delay caused by waiting for the common IA window, pure beam search efficiency could be compared using $\eta$. The evolution of $\eta$ against training episodes for the proposed approach and the methods in [6] and [8] are shown in Fig. 5. The work in [8] requires 7 beam (SSB) transmissions, 


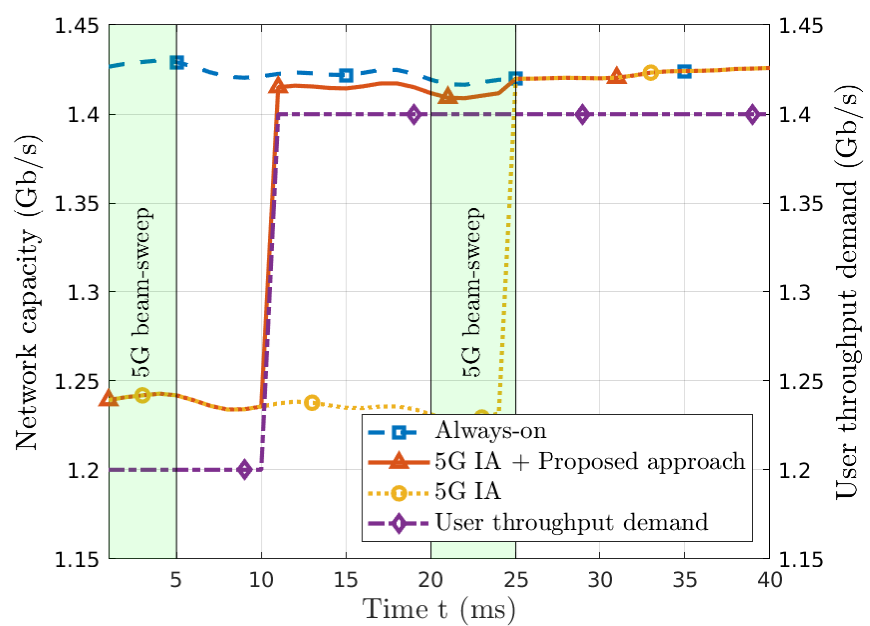

Fig. 4: Network capacity of 5G IA with and without the proposed method under dynamic user throughput demand.

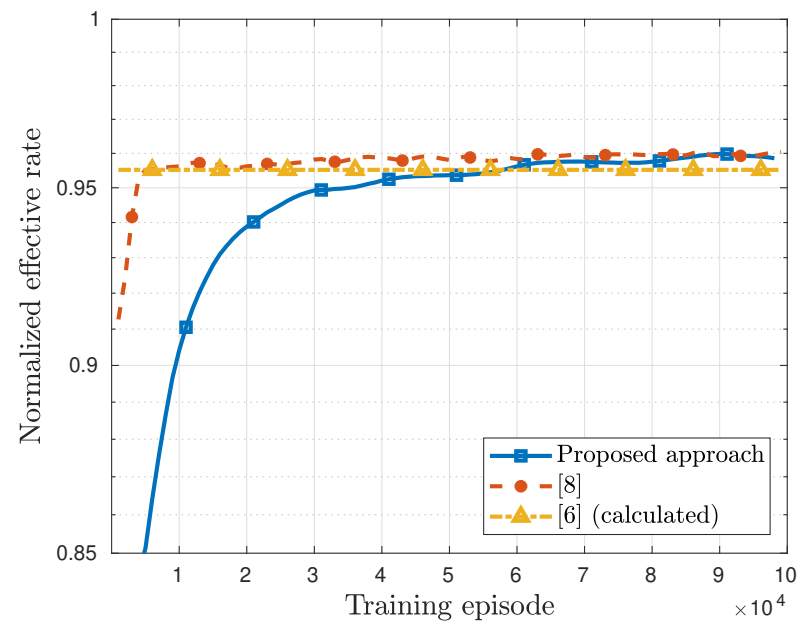

Fig. 5: Normalized effective rate.

and therefore, $T_{I A}$ is $0.56 \mathrm{~ms}$. The method in [6] claims to use $30 \%$ fewer beam transmissions compared to the exhaustive case which transmits all 16 beams. Therefore, $T_{I A}$ for [6] is calculated to be $0.896 \mathrm{~ms}$. An upper bound of the effective rate for the method in [6] is considered by assuming its beam selections are always optimal. The proposed approach only requires a small amount of data from the CPU and $T_{I A}$ for this wired transmission is estimated to be $0.01 \mathrm{~ms}$. Other overheads are ignored. The DCB of the proposed approach takes longer to learn compared to [8] due to the complex nature of the approximated mapping function. However, with adequate training, the proposed approach provides similar performance to that of [8] and the upper bound for [6].

The key advantage of the proposed approach is that it does not have to wait for the common IA window to identify the serving beam. Whereas the other approaches like [6] and [8] have to wait for this common IA window causing a significant additional delay on top of their respective $T_{I A}$ values.

\section{CONClusion}

In this paper, we have proposed a neighbor-aided approach based on DCB to provide instantaneous IA to APs waking up from a sleep mode. First, we have defined the function which maps beam choices made by the neighbors of an AP to it's beam codebook. Then, we have used a deep learning-based contextual bandit approach to solve the mapping problem. Finally, we have carried out simulations using realistic channels generated using a ray-tracing tool. The results show that the DCB model can successfully learn the mapping function and provide instantaneous beam prediction to aid current IA schemes. This work, therefore, presents a faster way to provide IA using neighbor information for APs in AP-dense mmWavebased UC networks.

\section{REFERENCES}

[1] Qualcomm, "The 1000x Mobile Data Challenge," White paper, no. November, pp. 1-38, 2013. [Online]. Available: https://www.qualcomm.com/media/documents/files/1000x-mobiledata-challenge.pdf

[2] I. K. Jain, R. Kumar, and S. S. Panwar, "The Impact of Mobile Blockers on Millimeter Wave Cellular Systems," IEEE Journal on Selected Areas in Communications, vol. 37, no. 4, pp. 854-868, 2019.

[3] T. Van Chien, E. Bjornson, and E. G. Larsson, "Joint power allocation and load balancing optimization for energy-efficient cell-free massive mimo networks," IEEE Transactions on Wireless Communications, vol. 19, no. 10, pp. 6798-6812, 2020.

[4] M. Feng, S. Mao, and T. Jiang, "BOOST: Base station on-off switching strategy for green massive MIMO HetNets," IEEE Transactions on Wireless Communications, vol. 16, no. 11, pp. 7319-7332, 2017.

[5] Technical Specification Group Radio Access Network, "3GPP TR 38.912: Study on New Radio (NR) access technology-Rel.16," 3GPP, Tech. Rep., 2020.

[6] I. Aykin and M. Krunz, "Efficient Beam Sweeping Algorithms and Initial Access Protocols for Millimeter-Wave Networks," IEEE Transactions on Wireless Communications, vol. 19, no. 4, pp. 2504-2514, 2020.

[7] J. Sung and B. L. Evans, "Compressed-Sensing based Beam Detection in 5G NR Initial Access," in IEEE International Workshop on Signal Processing Advances in Wireless Communications, may 2020, pp. 1-5.

[8] T. S. Cousik, V. K. Shah, T. Erpek, Y. E. Sagduyu, and J. H. Reed, "Deep Learning for Fast and Reliable Initial Access in AI-Driven 6G mmWave Networks," arXiv preprint, 2021. [Online]. Available: http://arxiv.org/abs/2101.01847

[9] GSMA, "5G TDD Synchronisation," Tech. Rep. April, 2020. [Online]. Available: https://www.gsma.com/spectrum/wpcontent/uploads/2020/04/3.5-GHz-5G-TDD-Synchronisation.pdf

[10] M. R. Akdeniz, Y. Liu, M. K. Samimi et al., "Millimeter wave channel modeling and cellular capacity evaluation," IEEE Journal on Selected Areas in Communications, vol. 32, no. 6, pp. 1164-1179, 2014.

[11] REMCOM, "Wireless insite reference manual," 2008.

[12] R. W. Heath, N. Gonzalez-Prelcic, S. Rangan, W. Roh, and A. M. Sayeed, "An Overview of Signal Processing Techniques for Millimeter Wave MIMO Systems," IEEE Journal on Selected Topics in Signal Processing, vol. 10, no. 3, pp. 436-453, 2016.

[13] M. Alrabeiah and A. Alkhateeb, "Deep Learning for mmWave Beam and Blockage Prediction Using Sub-6 GHz Channels," IEEE Transactions on Communications, vol. 68, no. 9, pp. 5504-5518, 2020.

[14] W. Chu, L. Li, L. Reyzin, and R. E. Schapire, "Contextual bandits with linear Payoff functions," Journal of Machine Learning Research, vol. 15, pp. 208-214, 2011.

[15] R. S. Sutton and A. G. Barto, Reinforcement Learning: An Introduction, 2nd ed. MIT Press, 2018.

[16] V. Mnih, K. Kavukcuoglu, D. Silver et al., "Human-level control through deep reinforcement learning," Nature, vol. 518, no. 7540, pp. 529-533, feb 2015 .

[17] Technical Specification Group Radio Access Network, "3GPP TR 38.213: NR Physical layer procedures for control," 3GPP, Tech. Rep., 2020. 